\title{
Incidental findings: the time is not yet ripe for a policy for biobanks
}

\author{
Jennifer Viberg ${ }^{\star,}$, Mats G Hansson ${ }^{1}$, Sophie Langenskiöld ${ }^{2}$ and Pär Segerdahl ${ }^{1}$
}

Incidental findings (IFs) are acknowledged to be among the most important ethical issues to consider in biobank research. Genome-wide association studies and disease-specific genetic research might reveal information about individual participants that are not related to the research purpose, but may be relevant to those participants' future health. In this article, we provide a synopsis of arguments for and against the disclosure of IFs in biobank research. We argue that arguments that do not distinguish between communications about pathogenic conditions and complex genetic risk for diseases fail, as preferences and decisions may be far more complex in the latter case. The principle of beneficence, for example, often supports the communication of incidentally discovered diseases, but if communication of risk is different, the beneficence of such communication is not equally evident. By conflating the latter form of communication with the former, the application of ethical principles to IFs in biobank research sometimes becomes too easy and frictionless. Current empirical surveys of people's desire to be informed about IFs do not provide sufficient guidance because they rely on the same notion of risk communication as a form of communication about actual health and disease. Differently designed empirical research and more reflection on biobank research and genetic risk information is required before ethical principles can be applied to support the adoption of a reasonable and comprehensive policy for handling IFs.

European Journal of Human Genetics (2014) 22, 437-441; doi:10.1038/ejhg.2013.217; published online 25 September 2013

A much discussed problem associated with biobank research is the return to participants of incidental findings (IFs): 'a finding concerning an individual research participant that has a potential health or reproductive importance and is discovered in the course of conducting research but is beyond the aims of the study.' ${ }^{1}$ How should such information be handled ethically responsibly in genome-wide association studies and disease-specific genetic research?

In this paper, we argue that the discussion up until now has neglected a distinction that should be held in the forefront of the discussion, especially concerning genetic biobank research: the distinction between an incidentally discovered disease and an incidentally discovered increased genetic risk for disease of unclear predictive value. Biobank research and rapidly increasing studies in genomics, proteomics, and nutrigenomics continue to identify many genes and biomarkers associated with risk of disease. Genetic testing for monogenic disorders are well established in health services, but little is yet known of the best way to handle complex risk information associated with multifactorial disorders in which the predictive importance of individual elements - genetic, epigenetic, or environmental - will differ for different individuals. The value of being informed about an incidentally discovered genetic risk (be it inherited or caused by a virus) is therefore much more difficult to ascertain than that for an incidentally discovered pathogenic condition revealed, for example, in a brain imaging study.

The aim of this paper is to exhibit the absence of a distinction between disease and complex genetic risk for disease in the discussion, and to show how the arguments therefore fail to address the more complex kinds of IFs that increasingly arise in biobank research. Further research should be conducted before the arguments can be considered conclusive.
Disease risks can be discovered also in imaging studies, of course, a blood vessel with thin walls can imply an increased risk for stroke. Our focus in this paper, however, is on genetic biobank research, where IFs increasingly concern multifactorial risks for disease having both genetic and environmental dimensions, which we believe introduce complications that so far have not been addressed.

\section{SYNOPSIS OF THE ARGUMENTATIVE FIELD}

We will not conduct a complete literature review of the arguments that have been used in ethical discussions of IFs in biobank research, but will only chart the most important kinds of arguments that have been used to discuss the subject in order to show how the distinction mentioned above is downplayed or neglected.

\section{ARGUMENTS FOR DISCLOSURE}

\section{Disclosure is beneficent for individuals}

A common normative statement in the discussion is that disclosure should be an option for participants because it will maximize their benefit and minimize harm if participants receive timely risk information. Under this argument, conditions have been formulated in which IFs are likely to impart benefit to the participant and therefore should be disclosed. If the genetic information reveals significant risk of a condition likely to be life threatening, can be used to avoid or to ameliorate a condition likely to be grave, or can be used in reproductive decision making, the information is held to be beneficent and appropriate for return. ${ }^{1,2}$ It has been emphasized that IFs are beneficent and should be returned if they are analytically valid, clinically significant, and actionable. ${ }^{3}$

\footnotetext{
${ }^{1}$ Department of Public Health and Caring Sciences, Centre for Research Ethics \& Bioethics, Uppsala University, Uppsala, Sweden; ${ }^{2}$ Department of Public Health and Caring Sciences, Health Economics, Uppsala University, Uppsala, Sweden

*Correspondence: J Viberg, Department of Public Health and Caring Sciences, Centre for Research Ethics \& Bioethics, Uppsala University, Box 564, Uppsala SE-751 22, Sweden. E-mail: jennifer.viberg@crb.uu.se
} 


\section{Disclosure promotes autonomy}

The principle of respect for persons, or respect for autonomy, is used as a premise in another argument for disclosure. If people get important information in time, they can change their lives and therefore be more autonomous; by knowing, individuals can take control over their lives and direct it as they wish. Respect for persons includes respect for participants' self-determination and therefore also for their need to have information relevant to their health and wellbeing, and thus motivates disclosure. ${ }^{4}$ In a similar vein, it is argued that if results have clear clinical use, or are relevant to life decisions, there is an obligation based on respect for persons to disclose them. Further, it is argued that it would be paternalistic to protect participants from potential anxiety instead of letting them know what is known about them. ${ }^{5}$

\section{Reciprocity requires disclosure}

Reciprocity between researchers and participants can be maintained by giving participants something in return for the participants' donation, in this case individual research results. It has been emphasized that participants' contribution to research cannot be assumed to be purely altruistic with no expectations of some personal gain, including knowledge, in return. ${ }^{6}$ This argument holds that people deserve something in return for their contribution to an enterprise or to society. ${ }^{4}$ It may also be argued that research would benefit from disclosing individual research results to participants; as offering something in return might motivate participation, the offering of individual findings could be useful in recruiting and retaining research participants. ${ }^{7}$ Reciprocity may also promote trust between researchers and research participants. ${ }^{3}$

\section{Return of IFs accords with participants' wishes}

Empirical surveys show that many people want to receive individual results. ${ }^{7,8}$ People do not consider their contribution as a gift, but participate in research with the expectation of getting something in return. ${ }^{9}$ Another study of public preferences suggests that people want to receive individual research results and that they believe that researchers have a duty to inform participants about mutations in their genes. This wish to receive individual results is typically motivated by the potential of such information to be used to improve health through changing health-related behaviors, getting treatment, or preventing disease. Some informants in this survey maintained that findings about them actually belong to them as a matter of ownership. ${ }^{10}$

\section{ARGUMENTS AGAINST DISCLOSURE}

\section{Practical issues make disclosure unfeasible}

It is sometimes claimed that it would be too time consuming and costly to contact research participants, and that disclosure would therefore inhibit important research. ${ }^{11}$ It has also been argued that variability in biobanks and practical implementation issues (biobanks vary in scale, biobank projects take a variety of forms, samples may be drawn from healthy participants or from those with disease) make it difficult to have a 'one-size-fits-all' approach to disclosing individual results. Moreover, the return of individual results to participants requires that biobanks retain links to identifying information, which implies the risk of breaching confidentiality. The issue of who should be responsible for recontacting participants, whose samples may be involved in many projects over a long period of time, is also unresolved. ${ }^{12}$
Another argument posits that if participants have a right to know about IFs, they must also have a corresponding right not to know. ${ }^{13}$ If participants have the right to choose whether or not to know, this option should therefore be prominent on the consent form. ${ }^{14}$ This brings up further practical issues about how informed choices can be made about disclosing IFs, as by definition not even the researchers know what kinds of IFs may be found.

Another practical argument against disclosure is that it is virtually impossible to identify such findings in much biobank research. Cho ${ }^{15}$, for example, mentions that it can be difficult to distinguish IFs from other findings in genetic and genomic research because the research question can be very open-ended and descriptive. Virtually nothing (or everything) is 'incidental' because the research question rather is like an imperative to find complex patterns, the components of which may not be known at the outset.

\section{Disclosure can harm participants}

Disclosure of IFs can be harmful to participants if it is not valid or if no treatment can be offered. If participants cannot or do not know how to respond to IFs, they may suffer anxiety. ${ }^{16,17}$ Returning findings can have negative consequences for both biobanks and participants. Procedures must be in place to ensure that the analyzed sample is actually from the person it is believed to be from. Participants can be harmed by receiving risk information that does not apply to them. Such safety demands are lower in exploratory biobank studies than in biobanks used for clinical trials. Their results are therefore less trustworthy on an individual level. Participants also risk being harmed by being informed about and acting on IFs whose quality, accuracy, clinical utility, or even origin is uncertain. ${ }^{12}$ Ensuring the same quality in explorative studies as is required for clinical trials may arguably be too expensive for the biobank systems.

It is further important to consider that giving participants information about IFs blurs the distinction between research and healthcare, a confusion that resembles the therapeutic misconception. ${ }^{17,18}$ The therapeutic misconception is held by individuals who believe that they receive care when they function as research participants.

Randomization and other aspects of the scientific method, however, prohibit the application of personal care. ${ }^{19}$ The therapeutic misconception is traditionally discussed in connection with clinical trials. When IFs in biobank research are seen as a basis for decisions about treatment, however, this can create expectations among those who donate samples that resemble the therapeutic misconception. Receiving their results back may encourage participants to assume that the research was carried out for their own personal benefit. If participants are encouraged to expect individual results, they may think of their research participation as akin to receiving some form of care and they may expect treatment for risks or conditions suggested by their results. If, however, they do not receive further information and they are not recontacted, they will tend to assume that all is well, which could be also harmful rather than helpful.

A further aspect of biobank research that can cause harm if IFs are returned is that samples can be used in several studies. It can be disturbing and irrelevant for participant to receive a call, many years later after the donation of the DNA sample, of potentially healthimportant information. People might not know that research still is ongoing. They may even have forgotten that they donated a sample, as donation is not as concrete and memorable as undergoing functional magnetic resonance imaging or computed tomography. ${ }^{20}$ 


\section{The relationship does not create a duty}

Another concern is that researchers do not have the same close and individual relationship to the participants as doctors have to their patients, and are not trained in the counseling skills necessary to returning individual results properly. ${ }^{12}$ This is more than a merely practical difficulty. If there is no doctor-patient relationship between researcher and participants, there is also no duty of disclosure. ${ }^{14,17,18}$

\section{Disclosure can harm research and prevent research from doing good}

Another line of argument against the disclosure of IFs focusses on the need to distinguish between research and care. ${ }^{21}$ Unlike the therapeutic misconception mentioned above, this argument focusses not on the participants' perceptions, but on the concern that if research begins to be organized similar to healthcare, it will harm the aims of research. The biomedical principles of beneficence and nonmaleficence (do good and do not harm) may be misapplied if the distinction between research and care is neglected. Doing good in healthcare and doing good in research have different meanings, and the different contexts produce different rights and duties. It is argued that the beneficence sought in research must be understood on the collective level and not on the individual level. Returning IFs to individual participants would be seeking to do good in an individualized fashion that is inappropriate for research. And because participants contribute to research, not to healthcare, it would be wrong not to maximize beneficence on the collective level appropriate to research. ${ }^{17,18,22}$ There is, moreover, a risk that disclosure of 'preliminary results from ongoing epidemiological studies may jeopardize the scientific validity of the study because of changes in behavior or selective dropouts.'17

The clinical ethos, then, cannot be transferred directly to the research setting because research has a purpose different to that of care. ${ }^{17,18}$ Forsberg et al point out the similarity between donating money to the Red Cross and donating a sample to biobank research. From the donor's point of view, the moral duty of the Red Cross is to ensure that the donation leads to as much of the intended good as possible, and not to inform individual donors about the results of the donation. ${ }^{17}$

\section{SUGGESTED POLICIES DO NOT ADDRESS THE COMPLEXITY OF GENETIC RISK INFORMATION}

As shown, the main arguments for disclosure focus on the possibility that disclosure can be beneficent to participants' health if the information is validated, has clinical utility, and is actionable. Disclosure can also promote autonomy, support reciprocity, and satisfy participants. The arguments against disclosure of IFs are that it is not practically feasible, it can be harmful to participants, and there is no relationship between researcher and participant that creates such a duty. Disclosure can also harm research.

The arguments for disclosure depend very much on the hypothetical possibility that knowing genetic risk information might be beneficent for participants. In brief, if the information is valid and useful, participants who want to know should be informed. Here are some examples of how this is expressed:

'If results can enhance treatment and care, there is an ethical imperative to offer feedback. ${ }^{5}$

'IFs with confirmed clinical utility where there is the possibility of treatment or prevention should be disclosed, with exceptions. ${ }^{23}$
Knoppers et $a l^{3}$, as mentioned before, argued that IFs should be returned if they reveal material risks that have:

(1) analytical validity,

(2) clinical significance, and

(3) actionability.

If results can enhance treatment, if they concern a material risk, if they have clinical utility, if they are life-saving - then they should be disclosed. It may seem that no one could object to these proposals, as they condition disclosure to what clearly would be beneficial for the participant. However, we intend to show that these properties are less self-evident in the case of multifactorial risk information.

The prevalence of provisos in the argumentation shows up in an unexpected way in a systematic review of arguments by Christenhusz et l. $^{23}$ According to this review, 'the strongest reason in favor of disclosure of an IF is its confirmed clinical utility and the possibility of treatment or prevention.' The problem is that this is hardly an argument for disclosure of genetic risk information of unclear predictive value, although it is an oft-repeated prerequisite for the disclosure of genetic information.

\section{IDEALIZED CONDITIONS FOR THE APPLICATION OF ETHICAL PRINCIPLES}

Why are there so many provisos in the arguments for disclosure of IFs in biobank research? We believe these conditions reveal a tendency to conflate genetic risk information with the kind of IFs that are more characteristic of imaging studies, for example, in which the IF may be a tumor - information that obviously should be communicated to the participant.

'Comparison to IFs in imaging studies is instructive,' Wolf et al write, but in our view the analogy can introduce problems. Disease risks can of course be discovered also in imaging studies (such as a blood vessel with thin walls implying an increased risk of stroke), and it seems that the provisos work to handle such cases. Very few would deny that those types of IFs, likely to occur with some frequency in imaging studies, should be returned. Relevant ethical principles are applicable and support disclosure; but do the conditions of analytical validity, clinical significance, and actionability imply the same straightforward beneficence to the disclosure of complex genetic risk information of unclear predictive value? One could easily be led to think so if one believes that genetic risk information reveals possible futures. In this view, although an actual tumor (or a fragile blood vessel) would not be discovered in biobank research, the possibility of a tumor developing in the future might be discovered and the possible tumor treated before it began. But can genetic information about cancer risk really be understood to reveal 'possible' tumors that can be treated before they 'actually' develop?

In another discussion, about informed consent and informational privacy, Manson and $\mathrm{O}^{\prime} \mathrm{Neil}^{24}$ point out that 'possessing genetic information is not like possessing a crystal ball, and future facts are not 'contained' within DNA.' If they are right, the discussion we surveyed in this article builds on a questionable analogy. Between genes and multifactorial diseases like diabetes, cardiovascular disease, and dementia, there are complex processes involving both genes and environment. The disease depends not only on deviation in several, rather than single, genes but also on interaction with environmental factors such as diet, exercise, and smoking. The information given to participants will be an expression of a risk that is dependent on not only on various environmental factors but also on the penetrance of the disease (ie, how likely it is that a particular genetic defect will be 
expressed and actually lead to symptoms). The meaning of the risk information also depends on the level of accuracy of the analytical test, as described before.

Actionability, then, has a different meaning when applied to genetic risks of unproven predictive value rather than to an accidentally discovered tumor, a fragile blood vessel, or a genetic risk associated with dominating genes with high penetrance. By downplaying such differences, these conditions are idealized as if inherently beneficial, regardless of whether we are discussing diseases and easily identifiable conditions implying immediate risk, or complex and multifactorial disease risks. On closer examination, then, these conditions are not as realistic as they first appear, but seem to be posited to mirror the requirements for applying the principles. They do not provide sufficient friction for the real-life application of the principles and cannot be used to decide whether it would actually be beneficial to a participant to know the genetic risk information under discussion.

What determines the actual medical case history of a human life, if Manson and $\mathrm{O}^{\prime} \mathrm{Neil}^{24}$ are right, is a tremendously complex and variable interaction between genes and environment. This complexity is not reflected in the mantra of analytical validity, clinical significance, and actionability. There is therefore a need to move beyond this verbally constructed façade of beneficence and explore how genetic risk information can be perceived and evaluated in reality. Would people really want to receive genetic risk information of unproven predictive value?

\section{EMPIRICAL SURVEYS NEED TO TAKE THE COMPLEXITY OF GENETIC RISK INFORMATION INTO ACCOUNT}

A reason for disclosure emphasized also in discussions that take objections seriously is that 'empirical studies confirm that participants prefer to have genetic results returned to them, at least when the results are actionable and accurate. ${ }^{25}$ The problem that we want to address, however, is that the simplistic provisos reappear in these studies (and are repeated in arguments using these studies in favor of disclosure). $7,10,26,27$ The results of studies with such a design are predictable. If you ask people whether they would want information about an IF if the finding meets the conditions $x, y$, and $z$, and $x, y$, and $z$ make the finding sound beneficial to know, then they will probably answer in the affirmative. If we are right, however, genetic risk information has a complexity that makes it difficult to assess accuracy and actionability. For this reason, it is interesting to note that informants tend to change their attitude to individual genetic findings when they are informed about the actual nature of such findings in typical biobank research. ${ }^{7}$ A quantitative survey showed that patients, who can be assumed to know more about the complexity of multifactorial diseases, were slightly less strong in their preference for receiving individual research results and their opinions about the researchers' duty to inform than were a representative sample of the general (Dutch) population. ${ }^{8}$

This suggests that the methods of conducting empirical studies need to be changed and highlight the complexity of genetic risk information in the questions posed. Instead of asking potential participants whether they would want health relevant genetic information, to which they could hardly say no, they should be asked questions based on realistic presentations of risk information and what it means.

Survey data may be helpful in formulating research hypotheses on which preferences should guide policy making. However, surveys are not causal in nature. They can only show correlations of preferences. Moreover, they do not capture the trade-offs that respondents might make when facing complex decisions, for example, would they want to receive IFs if the risk were of unproven predictive value, had low penetrance, and would not result in symptoms until after perhaps another 30 years? Therefore, new methods are needed to capture what kinds of risk information participants truly prefer to receive, and their relative importance for participants.

\section{CONCLUSION}

IFs in genetic research, in genome-wide association studies as well as in disease-specific studies, need to be explored differently, both theoretically and empirically. Theoretically, the discussion needs to address the actual nature of genetic risk information and the complexity of the modern understanding of genetics. Arguments for disclosure should not rely on repeating beneficial-sounding provisos that do not reflect this complexity. New empirical studies need to be designed in which genetic risk of unproven predictive value is described to informants, and not as a revelation of future conditions that can be treated before they are manifest. Informants' responses to offers of genetic risk information are relevant only if they understand what that information really is and how it differs from information about disease or immediate disease risk that can be obtained from imaging studies or other tests.

This article is not meant as another argument against the disclosure of IFs in biobank research. Rather, our point is that existing arguments and empirical evidence fail to address some of the most relevant properties of the IFs under discussion, namely, unproven predictive value. Perhaps when this complexity and uncertainty is taken into account in the future discussions, theoretically as well as in empirical studies, support for a policy of returning such findings can still be found. However, we are not there yet.

\section{ACKNOWLEDGEMENTS}

The research for this paper was made possible by funding from the IMI project BT-CURE (Grant agreement no. 115142-1), the EU Seventh Framework Programs RD-Connect, EuroTeam, BiobankCloud, and BBMRI-LPC, and the BBMRI.se infrastructure project financed by the Swedish Research Council.

1 Wolf SM, Lawrenz FP, Nelson CA et al: Managing incidental findings in human subjects research: analysis and recommendations. J Law Med Ethics 2008; 36: 219-248, 211

2 Wolf SM, Crock BN, Van Ness B et al: Managing incidental findings and research results in genomic research involving biobanks and archived data sets. Genet Med 2012; 14: 361-384.

3 Knoppers BM, Deschenes M, Zawati MH, Tasse AM: Population studies: return of research results and incidental findings Policy Statement. Eur J Hum Genet 2012; 21: 1-3.

4 Wolf SM, Paradise J, Caga-anan C: The law of incidental findings in human subjects research: establishing researchers' duties. J Law Med Ethics 2008; 36: 361-383, 214

5 Affleck P: Is it ethical to deny genetic research participants individualised results? $J$ Med Ethics 2009; 35: 209-213.

6 Quaid KA, Jessup NM, Meslin EM: Disclosure of genetic information obtained through research. Genet Test 2004; 8: 347-355.

7 Murphy J, Scott J, Kaufman D, Geller G, LeRoy L, Hudson K: Public expectations for return of results from large-cohort genetic research. Am J Bioeth 2008; 8: 36-43.

8 Meulenkamp TM, Gevers SK, Bovenberg JA, Koppelman GH, van Hylckama Vlieg A Smets EM: Communication of biobanks' research results: what do (potential) participants want? Am J Med Genet A 2010; 152A: 2482-2492.

9 Gaskell G, Gottweis H, Starkbaum J et al: Biobanks and European public opinion explaining heterogeneity. http://www.univie.ac.at/LSG/papers2011/LSG\%20Working\%20Paper.pdf. Accessed 23 January 2013.

10 Bollinger JM, Scott J, Dvoskin R, Kaufman D: Public preferences regarding the return of individual genetic research results: findings from a qualitative focus group study. Genet Med 2012; 14: 451-457.

11 Bledsoe MJ, Clayton EW, McGuire AL, Grizzle WE, O'Rourke P Zeps N Return of research results from genomic biobanks: cost matters. Genet Med 2012; 15: 103-105. 
12 Bledsoe MJ, Grizzle WE, Clark BJ, Zeps N: Practical implementation issues and challenges for biobanks in the return of individual research results. Genet Med 2012; 14: 478-483.

13 Kaye J, Boddington P, de Vries J, Hawkins N, Melham K: Ethical implications of the use of whole genome methods in medical research. Eur J Hum Genet 2010; 18: 398-403.

14 Ravitsky V, Wilfond BS: Disclosing individual genetic results to research participants. Am J Bioeth 2006; 6: 8-17.

15 Cho MK: Understanding incidental findings in the context of genetics and genomics. J Law Med Ethics 2008; 36: 280-285, 212.

16 Hens K, Nys H, Cassiman JJ, Dierickx K: The return of individual research findings in paediatric genetic research. J Med Ethics 2011; 37: 179-183.

17 Forsberg JS, Hansson MG, Eriksson S: Changing perspectives in biobank research: from individual rights to concerns about public health regarding the return of results. Eur J Hum Genet 2009; 17: 1544-1549.

18 Solberg B, Steinsbekk K: Managing incidental findings in population based biobank research. Nor Epidemiol 2012; 21: 195-201.

19 Appelbaum PS, Roth LH, Lidz CW, Benson P, Winslade W: False hopes and best data: consent to research and the therapeutic misconception. Hastings Cent Rep 1987; 17: 20-24.
20 Clayton EW: Incidental findings in genetics research using archived DNA. J Law Med Ethics 2008; 36: 286-291.

21 Meltzer LA: Undesirable implications of disclosing individual genetic results to research participants. Am J Bioeth 2006; 6: 28-30.

22 Fryer-Edwards K, Fullerton SM: Relationships with test-tubes: where's the reciprocity? Am J Bioeth 2006; 6: 36-38.

23 Christenhusz GM, Devriendt K, Dierickx K: To tell or not to tell? A systematic review of ethical reflections on incidental findings arising in genetics contexts. Eur J Hum Genet 2012; 21: 248-255.

24 Manson AC, O'Neill O: Rethinking Informed Consent in Bioethics. New York, NY, USA: Cambridge University Press, 2007.

25 Bredenoord AL, Kroes HY, Cuppen E, Parker M, van Delden JJ: Disclosure of individual genetic data to research participants: the debate reconsidered. Trends Genet 2011; 27: 41-47.

26 Beskow LM, Dean E: Informed consent for biorepositories: assessing prospective participants' understanding and opinions. Cancer Epidemiol Biomarkers Prev 2008; 17: $1440-1451$

27 Ruiz-Canela M, Valle-Mansilla JI, Sulmasy DP: What research participants want to know about genetic research results: the impact of "genetic exceptionalism". J Empir Res Hum Res Ethics 2011; 6: 39-46. 